\title{
BMJ Open Surgical aortic valve replacement in the era of transcatheter aortic valve implantation: a review of the UK national database
}

Marjan Jahangiri (D) , ${ }^{1}$ Rajdeep Bilkhu, ${ }^{2}$ Andrew Embleton-Thirsk, ${ }^{3}$ Hakim-Moulay Dehbi, ${ }^{3}$ Krishna Mani, ${ }^{1}$ Jon Anderson, ${ }^{4}$ Vassilios Avlonitis, ${ }^{2}$ Max Baghai, ${ }^{5}$ Inderpaul Birdi, ${ }^{6}$ Karen Booth, ${ }^{7}$ Amal Bose, ${ }^{8}$ Norman Briffa, ${ }^{9}$ Keith Buchan, ${ }^{10}$ Sunil Bhudia, ${ }^{11}$ Alex Cale, ${ }^{12}$ Indu Deglurkar, ${ }^{13}$ Shakil Farid, ${ }^{14}$ Leonidas Hadjinikolaou, ${ }^{15}$ Martin Jarvis, ${ }^{16}$ Seyed Hossein Javadpour, ${ }^{17}$ Reubendra Jeganathan, ${ }^{18}$ Manoj Kuduvalli, ${ }^{19}$ Kulvinder Lall, ${ }^{20}$ Jorge Mascaro, ${ }^{21}$ Dheeraj Mehta, ${ }^{13}$ Sunil Ohri, ${ }^{22}$ Prakash Punjabi, ${ }^{4}$ Rajamiyer Venkateswaran, ${ }^{23}$ Paul Ridley, ${ }^{24}$ Christopher Satur, ${ }^{24}$ Serban Stoica, ${ }^{25}$ Uday Trivedi, ${ }^{26}$ Afzal Zaidi, ${ }^{27}$ Patrick Yiu, ${ }^{28}$ Narain Moorjani, ${ }^{29}$ Simon Kendall, ${ }^{30}$ Nick Freemantle ${ }^{31}$

To cite: Jahangiri $M$, Bilkhu R, Embleton-Thirsk A, et al. Surgical aortic valve replacement in the era of transcatheter aortic valve implantation: a review of the UK national database. BMJ Open 2021;11:e046491. doi:10.1136/ bmjopen-2020-046491

- Prepublication history for this paper is available online. To view these files, please visit the journal online (http://dx.doi. org/10.1136/bmjopen-2020046491).

$\mathrm{RB}$ and $\mathrm{AE}-\mathrm{T}$ contributed equally.

Received 31 October 2020 Accepted 21 September 2021

Deck for updates

(c) Author(s) (or their employer(s)) 2021. Re-use permitted under CC BY-NC. No commercial re-use. See rights and permissions. Published by BMJ.

For numbered affiliations see end of article.

\section{Correspondence to}

Professor Marjan Jahangiri; marjan.jahangiri@stgeorges. nhs.uk

\section{ABSTRACT}

Objectives To date the reported outcomes of surgical aortic valve replacement (SAVR) are mainly in the settings of trials comparing it with evolving transcatheter aortic valve implantation. We set out to examine characteristics and outcomes in people who underwent SAVR reflecting a national cohort and therefore 'realworld' practice.

Design Retrospective analysis of prospectively collected data of consecutive people who underwent SAVR with or without coronary artery bypass graft (CABG) surgery between April 2013 and March 2018 in the UK. This included elective, urgent and emergency operations. Participants' demographics, preoperative risk factors, operative data, in-hospital mortality, postoperative complications and effect of the addition of CABG to SAVR were analysed.

Setting $27(90 \%)$ tertiary cardiac surgical centres in the UK submitted their data for analysis.

Participants 31277 people with AVR were identified. 19670 (62.9\%) had only SAVR and 11607 (37.1\%) had AVR +CABG.

Results In-hospital mortality for isolated SAVR was $1.9 \%$ (95\% Cl $1.6 \%$ to $2.1 \%$ ) and was $2.4 \%$ for AVR+CABG. Mortality by age category for SAVR only were: $<60$ years $=2.0 \%, 60-75$ years $=1.5 \%,>75$ years $=2.2 \%$. For SAVR+CABG these were; $2.2 \%, 1.8 \%$ and $3.1 \%$. For different categories of EuroSCORE, mortality for SAVR in low risk people was $1.3 \%$, in intermediate risk $1 \%$ and for high risk $3.9 \%$. $74.3 \%$ of the operations were elective, $24 \%$ urgent and $1.7 \%$ emergency/salvage. The incidences of resternotomy for bleeding and stroke were $3.9 \%$ and $1.1 \%$, respectively. Multivariable analyses provided no evidence that concomitant CABG influenced outcome. However, urgency of the operation, poor ventricular function, higher EuroSCORE and longer cross clamp and cardiopulmonary bypass times adversely affected outcomes.

\section{Strengths and limitations of this study}

This is a large study of consecutive participants who have undergone surgical aortic valve replacement \pm coronary artery bypass graft surgery in the UK, reporting contemporary outcomes.

- This study includes people of all age groups and risks factors, and elective as well as urgent and emergency operations.

- The results are of in-hospital mortality and complications and longer-term follow-up data are not available.

Conclusions Surgical SAVR \pm CABG has low mortality risk and a low level of complications in the UK in people of all ages and risk factors. These results should inform consideration of treatment options in people with aortic valve disease.

\section{BACKGROUND}

Aortic valve disease, especially aortic stenosis affects $5 \%$ of the population and $3.9 \%$ of those between the ages of 70 and 79 and nearly $10 \%$ of those above the age of $80 .^{1}$ Severe aortic stenosis when untreated has a risk of death of $50 \%$ at 2 years. $^{2}$ Conventionally the gold standard of treatment has been surgical aortic valve replacement (SAVR). However, the role of transcatheter aortic valve implantation (TAVI) has evolved in recent years. TAVI was first introduced in 2002, initially being performed in high risk inoperable patients. ${ }^{3}$ The original Placement of Aortic Transcatheter Valves (PARTNER) 
trial demonstrated a significant reduction in mortality, repeat hospitalisation and cardiac symptoms compared with inoperable patients who had only medical therapy. ${ }^{4}$ The original PIVOTAL study also demonstrated significantly higher survival at 1 year in high-risk patients who underwent SAVR. ${ }^{5}$

The role of TAVI is being extended to lower risk and younger patients based on recent trials comparing SAVR with TAVI. ${ }^{6}$ Several studies suggest there has been a change in demographics and types of surgical valves used since the advent of TAVI. ${ }^{8-10}$ There has been a trend of increased use of tissue valves and a decrease in the use of mechanical valves in recent years. ${ }^{11}$ This may be due to the evolution of TAVI practice whereby younger patients can have a tissue valve with the view that they have a TAVI valve in the future when the tissue valve has deteriorated, so called valve-in-valve. ${ }^{1213}$

The series in the literature reporting outcomes of SAVR are generally unit based. ${ }^{914}$ Also, people with aortic valve disease are given information about the outcomes of SAVR which may be out of date and incorrectly extrapolated from smaller studies. There is a lack of contemporary national data to assess the outcomes of SAVR (mortality and complications), and to demonstrate the trend in use of prosthetic valves which would inform people with aortic valve disease better. There are some perceived complications of surgery that may be understood by referring general practitioners and cardiologists to be prohibitive risks for surgery.

In order to inform practitioners, people with aortic valve disease and the cardiac surgical community, we set out to examine the results of contemporaneous SAVR in a multicentre study of UK cardiac surgical units, in the era of TAVI. In addition, we summarise and interpret some of the more recent trials on the management of aortic valve disease.

\section{METHODS}

\section{Data}

This is an analysis of prospectively collected data of people who underwent SAVR \pm coronary artery bypass graft (CABG) surgery between April 2013 and March 2018 in the UK and Republic of Ireland. Anonymised data were submitted to the Society for Cardiothoracic Surgery of Great Britain \& Ireland for 27 of the 30 units and then stored in a secure database. This period was chosen to reflect fairly contemporary practice and also the data is submitted in March every year. The data are collected by each unit, validated and then submitted to the National Institute of Cardiovascular Outcome Research (NICOR). It took approximately 9 months to collect, validate and clean all the data. The outcome measures recorded are based on strict definitions provided by NICOR to provide uniformity.

Only participants who had had first time surgery, $\mathrm{SAVR} \pm \mathrm{CABG}$ were included. All participants immaterial of their risk for surgery, people of all age groups and risk factors were included. Those who required other concomitant procedures like replacement of parts of the aorta, aortic root enlargement, other valve procedures and redo surgery were excluded.

\section{Pre-operative risk factors and operative features}

Baseline demographic data; significant medical history such as diabetes, renal dysfunction, hypertension or stroke; predominant aortic valve pathology (stenosis or regurgitation or mixed valve disease) and preoperative left ventricular ejection fraction (LVEF) were collected. EuroSCORE is the risk stratification model used in the UK. Logistic EuroSCORE was collected as well as EuroSCORE II where available. The latter was only used since 2017 and therefore not available for all participants. Logistic EuroSCORE was divided into three categories: $<3 \%, 3 \%-6 \%,>6 \%$.

LVEF was divided into three categories: $\operatorname{good}(\mathrm{EF}>50 \%)$, moderate $(\mathrm{EF} 30 \%-50 \%)$ and poor $(\mathrm{EF}<30 \%)$. Transient ischaemic attack was defined as any neurological symptoms lasting $<24$ hours. Stroke was defined as new neurological dysfunction persisting $>24$ hours. Operative data including operative urgency: elective, urgent and emergency/salvage were recorded. Elective was defined as when the person was admitted from home, urgent meaning that the person was admitted with an urgent condition and required surgery during the same hospital admission, emergency and salvage meaning that surgery was required within 24 hours of admission and/or the person was in extremis. Other parameters including cardiopulmonary bypass (CPB) time, cross clamp time (CCT), type of valve implanted as well as concomitant CABG surgery performed were also collected.

\section{Postoperative outcomes}

Postoperative complication data were collected with the main focus being in-hospital mortality, new stroke, return to theatre for bleeding, deep sternal wound infection and duration of postoperative hospital stay.

\section{Statistical analysis}

Once the records for all participants were collated and the data cleaned, each factor was summarised using descriptive methods. Categorical variables are presented as $\mathrm{N}$ $(\%)$ and continuous variables are presented as median (IQR). New strokes were recoded to be either no stroke or any cerebrovascular accident (transient or permanent). The natural $\log$ of postoperative length of stay (days) was used due to positive skewed distribution of this variable. Univariate models were used, logistic regression for binary outcomes and linear regression for continuous outcomes, to assess the impact of the key explanatory variables. In these models, a two-tailed $\mathrm{p}<0.05$ was considered significant. The population analysed included all the participants with data collected, with results checked in the subset who had SAVR only (without CABG). Building on this, a multivariable model with all key variables in the model to assess which had the most impact on each of 


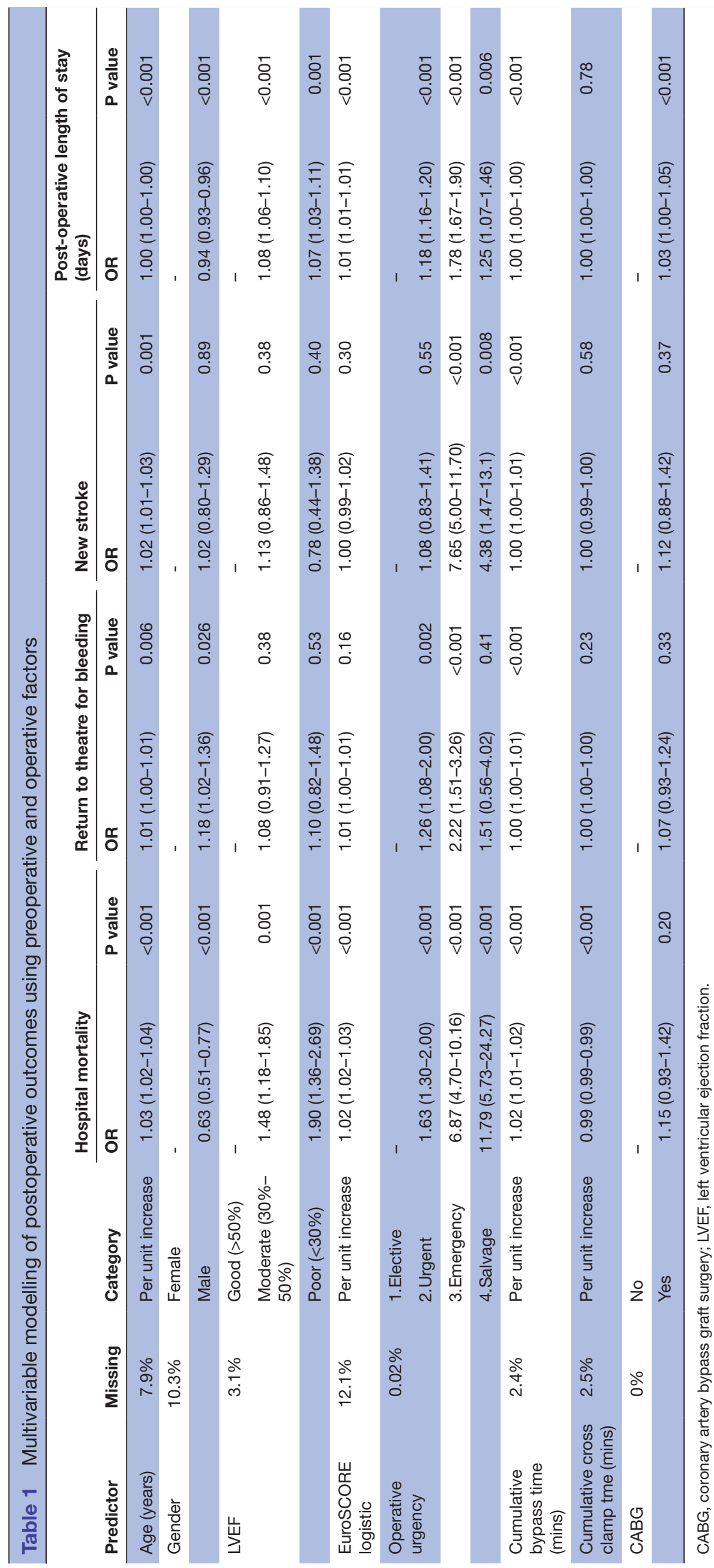

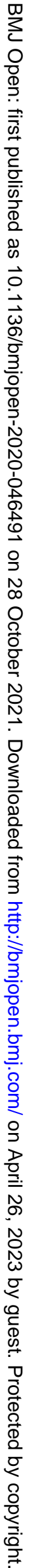


the outcomes was created. Stata/MP V.15.1 (StataCorp) was used for all analyses. Multiple imputation of missing data was not performed. The missingness was mostly negligible. There was no missing mortality and the data is shown in table 1 .

\section{Patient and public involvement}

Patients and public were involved in the original design of the database.

\section{RESULTS}

\section{Descriptive analysis}

In total 31277 patients were included. Of these, 19670 $(62.9 \%)$ had only SAVR and $11607(37.1 \%)$ had SAVR +CABG. There were $14.4 \%$ below the age of 60 , $46.9 \%$ between 60 to $75 \%$ and $36.7 \%$ older than 75 years with $7.9 \%$ missing age data. There were 1.9 times more males than females (10.3\% missing).

Regarding preoperative risk factors, $75.2 \%$ had good LVEF, $17.3 \%$ had moderate and $4.3 \%$ had poor LVEF. $74.3 \%$ of the operations were elective, $24 \%$ were urgent and $1.7 \%$ were emergency or salvage operations.

Logistic EuroSCORE had a median of 1.83 with an IQR of $0.06-6.0$. In total, $50 \%$ of patients were classified as low risk $(<3 \%), 16 \%$ as medium risk $(3-6 \%)$ and $22 \%$ high risk $(>6 \%) .3792$ patients $(12.1 \%)$ were missing data. The median EuroSCORE II was 1.95 (IQR 0.67-4.8) although with $56.5 \%$ with missing data, as this was introduced into the database in 2017. The median CPB time was $104 \mathrm{~min}$ (IQR 82-135) and CCT was $79 \mathrm{~min}$ (IQR 61-101).

For valve implant replacement type, $70 \%$ had a tissue valve, $12.2 \%$ a mechanical valve and $0.2 \%$ had homograft or autograft valves. For $17.3 \%$ the entry for valve type was unclear. The ratio of mechanical implant to bioprosthetic implant use has remained stable over time.

Overall mortality was $2.4 \%$ (95\% CI $2.2 \%$ to $2.6 \%$ ) and mortality for isolated SAVR for all participants was $1.9 \%$ (95\% CI $1.6 \%$ to $2.1 \%$ ). The mortality figures analysed for different age ranges and for categories of EuroSCORE are shown in table 2.

\begin{tabular}{|c|c|c|c|}
\hline Euroscore & $\mathrm{N}(\%)$ & $\begin{array}{l}\text { SAVR+CABG } \\
\text { (mortality \%) }\end{array}$ & $\begin{array}{l}\text { SAVR } \\
\text { (mortality \%) }\end{array}$ \\
\hline$<3 \%$ & $15619(50.0)$ & 2.0 & 1.3 \\
\hline $3 \%-6 \%$ & $5020(16.1)$ & 0.9 & 1.0 \\
\hline$>6 \%$ & 6846 (21.9) & 4.4 & 3.9 \\
\hline Age (years) & & SAVR+CABG & SAVR \\
\hline$<60$ & & 2.2 & 2.0 \\
\hline $60-75$ & & 1.8 & 1.5 \\
\hline$>75$ & & 3.1 & 2.2 \\
\hline
\end{tabular}

CABG, coronary artery bypass graft; SAVR, surgical aortic valve replacement.
Overall, $3.9 \%$ (3.6\% in SAVR only) of participants had resternotomy for postoperative bleeding or tamponade, $0.04 \%(0.06 \%$ in SAVR only) had reoperation for valvular problems (significant paravalvular leak and early endocarditis), $0.7 \%$ (0.6\% SAVR only) had reoperation for other cardiac problems, $0.2 \%$ ( $0.15 \%$ for SAVR only) had rewiring of sternum for sterile wounds and $0.14 \%(0.06 \%$ for SAVR only) had rewiring of sternum for infection. Transient ischaemic attack occurred in only $0.6 \%$ and $1.1 \%$ had a stroke (no missing data).

Median postoperative length of stay was 7 days (IQR: 6-11) in those with SAVR only and 8 days (IQR: 6-12) in all patients.

The number of bypass grafts was not analysed due to concerns about inconsistent reporting of data describing the number of grafts.

When comparing the two subsets of patients, the characteristics of those with SAVR alone were broadly similar to those with SAVR+CABG. In SAVR alone there were more people aged $<60$ years $(19.3 \%$ vs $6.2 \%)$, but less people were older than 75 ( $30.1 \%$ vs $43 \%)$. A higher proportion of those with SAVR+CABG were male $(68 \%$ vs $54 \%)$. Bypass time was an average of 37 min shorter and CCT 27 min in the SAVR alone group. Among those with only SAVR the mechanical valve usage was greater, at $16 \%$ vs $7 \%$.

\section{Regression analysis \\ Univariable}

Taken in isolation, all preoperative risk factors were associated with an increased odds of death, as was addition of CABG. The same pattern was observed when analysing the need for reoperation or surgery, with all explanatory variables indicative of a worse outcome without taking into account any others. For new stroke only age, EuroSCORE, operative urgency, EF and cumulative bypass and CCT affected a negative outcome (but not gender), as did CABG. All factors predicted a longer postoperative length of stay, including CABG.

As a sensitivity analysis, age categories were also assessed. When included as a continuous variable, age was significant both on its own and in all the multivariable models. All participants were categorised into $<60,60-75$ and $>75$ years of age. Those $60-75$ were at a lower odds of death in comparison to those $<60$ (OR $0.71,95 \%$ CI 0.53 to $0.95, \mathrm{p}=0.021$ ) with no difference in those $>75$ (OR $1.09,95 \%$ CI 0.82 to 1.45$)$ in the AVR alone group. These findings were different in the SAVR+CABG group, with no significant difference in those $60-75$ (OR $0.81,95 \%$ CI 0.64 to 1.03 ) but an increased risk in those $>75$ (OR 1.09, $95 \%$ CI 1.12 to $1.76, p=0.004$ ).

\section{Multivariable analyses}

Analysis of postoperative outcomes using multivariable models including all preoperative and operative factors are shown in table 1 . This demonstrated that age (OR 1.03 (95\% CI 1.02 to 1.04 ), $\mathrm{p}<0.001$ ), moderate EF (OR 1.48 (95\% CI 1.18 to 1.85 ), $\mathrm{p}<0.001$ ), poor EF (OR 1.90 
(95\% CI 1.36 to 2.69$), \mathrm{p}<0.001)$, logistic EuroSCORE (OR 1.02 (95\% CI 1.02 to 1.03 ), $\mathrm{p}<0.001$ ), urgent operation (OR 1.63 (95\% CI 1.30 to 2.00, $\mathrm{p}<0.001$ ), emergency surgery (OR 6.87 (95\% CI 4.70 to 10.16), $\mathrm{p}<0.001$ ) and longer CPB times affected mortality (OR 1.02 (95\% CI 1.01 to 1.02$), \mathrm{p}<0.001)$.

When all other variables were taken into account CABG was not significantly associated with an increase in the risk of death (OR 1.15 (95\% CI 0.93 to 1.42 ), $\mathrm{p}=0.20$ ).

Older age, (OR 1.01 (95\% CI 1.00 to 1.01 ), $\mathrm{p}<0.006$ ), longer CPB time (OR 1.00 (95\% CI 1.00 to 1.01 ), $\mathrm{p}<0.001$ ), urgent (OR 1.26 (95\% CI 1.08 to 2.00$)$, $\mathrm{p}<0.002)$ and emergency surgery (OR 2.22 (95\% CI 1.51 to 3.26$), \mathrm{p}<0.001)$ were significant factors in identifying people requiring return to theatre for bleeding. Again, CABG did not affect the odds of returning to theatre (OR 1.07 (95\% CI 0.93 to 1.24$), \mathrm{p}=0.33$ ).

Factors affecting stroke were age, (OR 1.02 (95\% CI 1.01 to 1.03 ), $\mathrm{p}<0.001$ ), emergency (OR 7.65 (95\% CI 5.00 to $11.70, \mathrm{p}<0.001)$ or salvage surgery (OR $4.38(95 \%$ CI 1.47 to 13.1$) \mathrm{p}=0.008)$ and CPB times (OR $1.00(95 \%$ CI 1.00 to 1.01$), \mathrm{p}<0.001)$. As in the other outcomes, addition of CABG did not affect the outcome (OR 1.12 (95\% CI 0.88 to 1.42 ), $\mathrm{p}=0.37$ ).

Age, male gender, moderate and poor EF, operative urgency, higher logistic EuroSCORE and cumulative bypass time significantly all affected postoperative length of stay.

\section{DISCUSSION}

This study reports contemporary results of SAVR and SAVR + CABG in the UK, reflecting real world practice, reporting an overall mortality of $1.9 \%$ and $2.4 \%$, respectively. We have shown a low mortality and complication rate for all comers following surgery in people requiring SAVR or SAVR + CABG. The complications were low with $3.9 \%$ resternotomy for bleeding, $0.04 \%$ reoperation for valvular problems and $1.1 \%$ stroke. Surprisingly, having accounted for other risk factors, addition of CABG did not adversely affect the outcomes.

The strengths of the study include its large number of participants, no exclusion of urgent and emergency/ salvage cases, and inclusion of all risk participants. The limitations are that three centres were unable to take part, possible coding errors in using large databases, lack of detailed echocardiographic data on valve annular size and presence or absence of preoperative infective endocarditis which can adversely affect outcomes. In addition, the results are in-hospital mortality and complications and the database lacks longer follow-up information.

Data from the current study are consistent with other large international studies. Data from the US Society of Thoracic Surgeons (STS) database demonstrated in-hospital mortality for isolated SAVR of $2.5 \%$ and incidence of stroke of $1.5 \% .{ }^{15}$ A recent analysis of the Japanese Cardiovascular Surgery database which assessed the outcomes of patients undergoing SAVR over a 8-year period has demonstrated a similar in-hospital mortality of $2 \% .{ }^{16}$ They also demonstrated a reduction in mortality over time, despite increasing surgical risk. The age of the patients in our study is lower than some of the trials of SAVR and TAVI. This is probably due to the selection criteria in these trials where older patients were selected.

We had set out to analyse the results of SAVR in the UK to inform practitioners treating people with aortic valve disease and inform people with this condition in an era where other therapies for management of aortic valve disease are evolving with expanding indications. Although the current study did not examine people who received TAVI, we discuss the various trials of SAVR and TAVI reported in the context of the literature and compare them with the results of the current study.

In tables 3-5, the demographics, procedural details and outcomes of the current study are compared with the respective sub-groups of the published trials. Table 5 shows low mortality and complication rate in the participants of this study following surgery in people who required SAVR or SAVR+ CABG. The trials comparing AVR and TAVI have enrolled and classified patients according to the risk of surgery, in particular the more recent trials. ${ }^{67}$ The most commonly used surgical risk stratification score is the STS risk score, although this scoring system has been validated in the US population. We have used EuroSCORE and shown that mortality is low in all categories of risk.

There are several recent trials comparing SAVR with TAVI. A meta-analysis of six of these trials performed by Barili et al reported that mortality was affected by the treatment modality with a time-varying effect: TAVI was related to better survival in the first months after implantation whereas, after 40 months, it was a risk factor for all-cause mortality. ${ }^{17}$ The NOTION trial, which compared outcomes of patients estimated to have low surgical risk who underwent either TAVI or SAVR demonstrated similar early mortality results, with mortality of $2.1 \%$ in TAVI group vs $3.7 \%$ in the SAVR group, $\mathrm{p}=0.380 .{ }^{18}$ The PIVOTAL trial of low-risk patients also reported similar results between those who underwent TAVI compared with SAVR, with early mortality of $0.5 \%$ in TAVI group and $1.3 \%$ in SAVR. $^{7}$ In addition, the 5-year results of the PARTNER 2 study, comparing TAVI versus SAVR in intermediate surgical risk demonstrated no significant difference in the incidence of death or stroke at 5 years following SAVR or TAVI. ${ }^{19}$ The mortality in the intermediate EuroSCORE risk category of the current study was $1.0 \%$ for SAVR only and $0.9 \%$ for SAVR + CABG. PARTNER 3, however, demonstrated significantly lower mortality in the TAVI group compared with SAVR ( $1 \%$ vs $2.5 \%, p=0.01)$ at 1 year. ${ }^{6}$ An observational study of 7618 patients comparing SAVR with TAVI at 5 years showed, however, that in a realworld population with low and intermediate risk, SAVR was associated with lower mortality and major adverse cardiac events, although this was with first generation TAVI devices. ${ }^{20}$ 


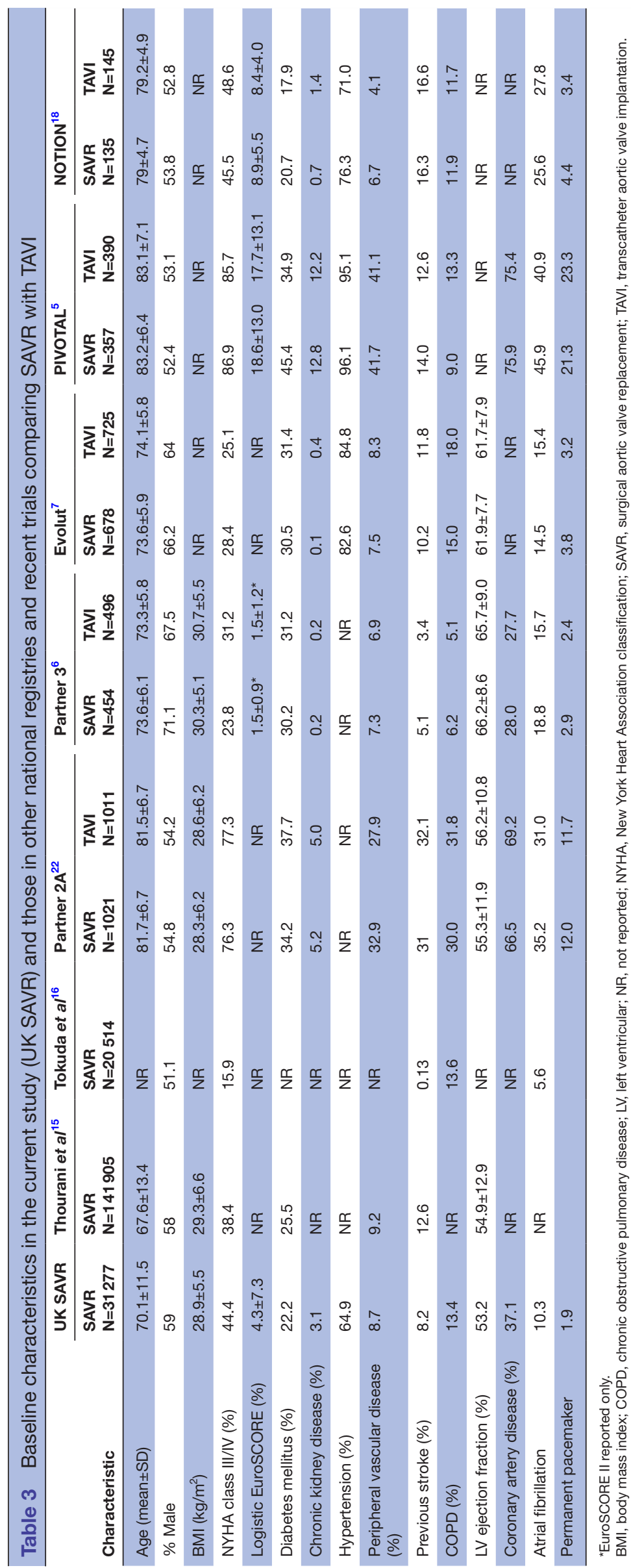



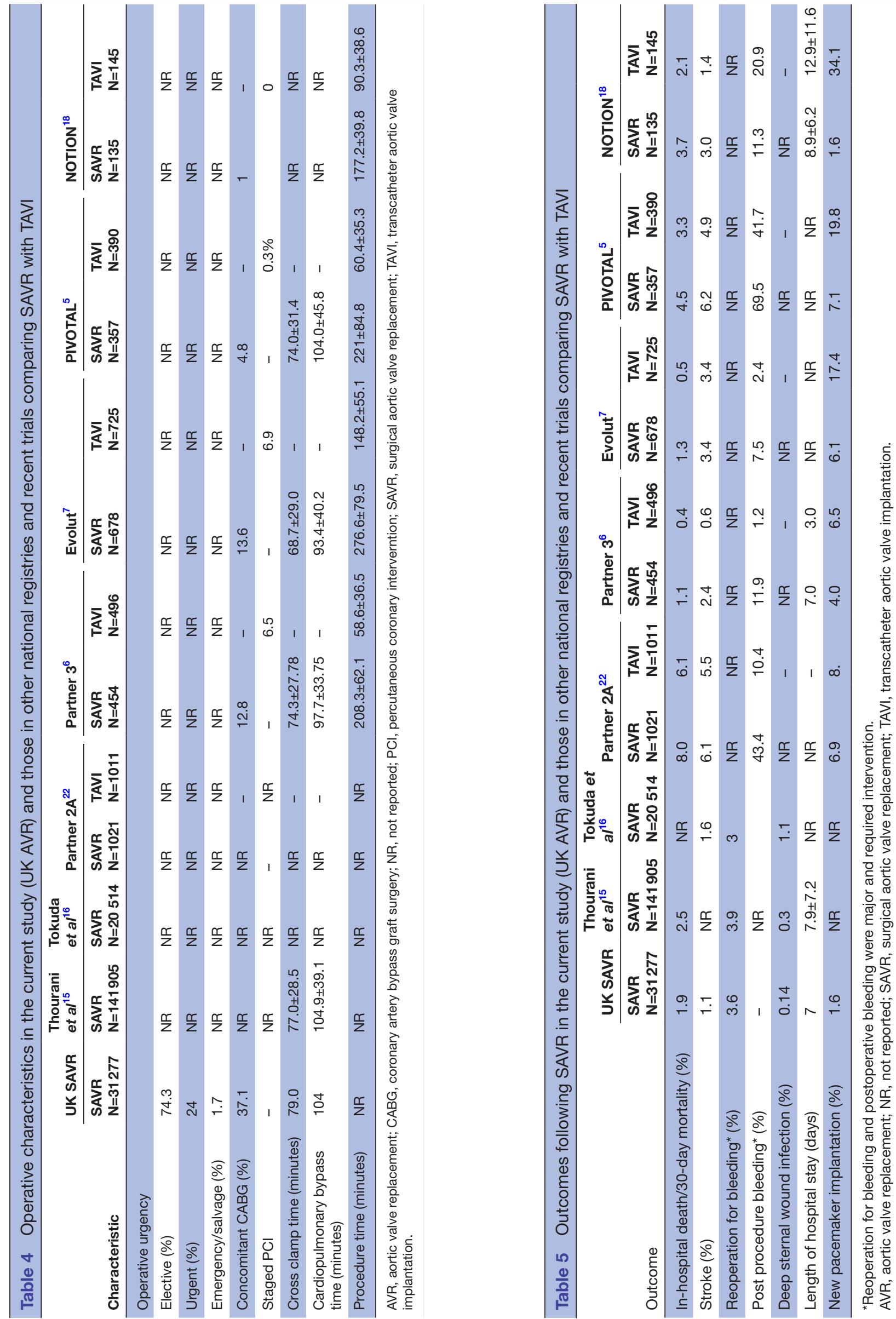
Role of coexistent coronary artery disease and its management

Sixty per cent of patients with aortic valve disease undergoing SAVR and $65 \%$ of those undergoing TAVI have coexisting coronary artery disease. ${ }^{21}$ In our series, $37 \%$ had coexistent coronary artery disease and underwent concomitant CABG. The addition of CABG did not adversely affect outcomes. The USA ${ }^{15}$ and Japanese ${ }^{16}$ series did not look into concomitant CABG. The percentage of concomitant CABG in our series is higher than the trials of SAVR/TAVI. This probably reflects the selection criteria in the latter. In PARTNER 2, although both groups had a similar number with coexistent coronary artery disease, $14.5 \%$ of the SAVR group had concomitant CABG compared with $3.9 \%$ of the TAVI group who had percutaneous intervention (table 4). ${ }^{22}$ SAVR may, therefore, be the preferred treatment modality in those with aortic stenosis and multivessel coronary artery disease requiring revascularisation.

Treatment of coronary artery disease in TAVI patients may require more than one hospital admission and can often result in incomplete revascularisation and its consequent increased morbidity and mortality. A meta-analysis by Sankaramangalam et al demonstrated that while there was no increase in mortality in patients with coronary artery disease who underwent TAVI at 30 days, there was a significant increase in mortality at 1 year following TAVI in these patients. ${ }^{23}$ The economic costs of readmission after TAVI have been demonstrated to be higher than in those who are readmitted after surgery and so untreated coronary disease which later requires readmission will have cost implications. ${ }^{24}{ }^{25}$ Surgery has the advantage of addressing all the pathology with one operation.

\section{Durability and choice of prosthetic valves}

In choosing the technique of treatment for aortic valve disease, life expectancy of the person and durability of the valve need to be considered. Ideally, the prosthetic valve should be durable for the person's lifetime. Both of these are related to person's age. In the UK, a 50-year-old woman has a life expectancy of 34 years and a 70-year-old man a life expectancy of 14 years. ${ }^{26}$

The durability of bioprosthetic valves is well documented in the surgical literature and is inversely proportional to person's age. Structural valve deterioration has been demonstrated to increase exponentially beyond 10 years following surgery. ${ }^{27}$ Considering the UK life expectancy, ${ }^{26}$ a 70 -year-old man has a $5 \%$ risk of reoperation and a 50-year-old woman has a $30 \%$ chance of needing a second operation.

Surgery has the advantage of offering the patient a mechanical or a bioprosthetic valve. The option of a mechanical valve which is only available in surgical SAVR should not be overlooked especially in younger people. In the current study, we have shown a fairly consistent ratio of tissue to mechanical valve use. However, the reported literature shows that the number of mechanical valve implantations has reduced in comparison to bioprosthetic valves. ${ }^{10}$ Mechanical valves are durable, with one group reporting $6.9 \%$ reintervention rate at 15 years vs $12.1 \%$ in those who underwent surgery with a bioprosthesis. ${ }^{29}$ For this reason, it has been the most commonly considered prosthesis in those under the age of 60 , as in our study where $60.2 \%$ of participants $<60$ years had a mechanical valve. Mechanical valves have the disadvantage of requiring anticoagulation, although, newer generations require a lower level of anticoagulation. ${ }^{30}$ Whilst mechanical valves are more durable, this has to be balanced against the greater risk of bleeding. ${ }^{29}$ At 15 years follow-up, Chiang et al also demonstrated no significant difference in survival and stroke between patients who underwent SAVR with mechanical vs bioprosthetic valve. ${ }^{29}$ Another group demonstrated in the $50-70$ years old cohort that survival at 5 years was higher in patients who had undergone SAVR with mechanical valve versus bioprosthesis and also demonstrated similar freedom from major bleeding events. ${ }^{31}$

\section{Bicuspid aortic valve and aneurysm of the aorta}

A significant number of patients requiring SAVR have bicuspid aortic valve, which has an incidence of $1 \%-2 \%$ in the general population and may present with aortic valve stenosis, regurgitation and ascending aortic aneurysm. The type of native aortic valve is not recorded in the database of our study. BAV may be present in up to $30 \%$ of patients undergoing SAVR. ${ }^{32}$ Bicuspid aortic valve anatomy, larger annular size, bulky and asymmetric leaflet calcification and dilated ascending aorta all pose technical challenges to TAVI which are not prohibitive risk factors for surgery. In fact, associated pathology of aneurysms of the aortic root and ascending aorta can be treated at the time of SAVR with little additional risk. ${ }^{33}$

European guidelines recommend discussing people with aortic valve disease in a multidisciplinary setting referred to as Heart Team, comprising of a surgeon, a non-interventional and an interventional cardiologist. ${ }^{34}$ This will allow the best treatment option to be put forward to the person.

\section{CONCLUSIONS}

SAVR with and without CABG has low mortality risk and a low level of complications in the UK in people of all ages and risk factors. Our study provides real-world experience of surgical results to improve understanding of the risks of surgery and decision making in a multidisciplinary team setting with heart team. The results of this study can be used by people with aortic valve disease, referring general practitioners, physicians, surgeons and policy-makers. Future studies need to address long-term follow-up including factors like quality of life which are currently not collected by the specialist centres.

\section{Author affiliations}

${ }^{1}$ Department of Cardiac Surgery, St George's Hospital, London, UK

'Department of Cardiac Surgery, St Thomas' Hospital, London, UK

${ }^{3}$ University College London Institute of Clinical Trials and Methodology, London, UK 
${ }^{4}$ Department of Cardiac Surgery, Hammersmith Hospital, London, UK

${ }^{5}$ Department of Cardiac Surgery, King's College Hospital, London, UK

${ }^{6}$ Department of Cardiac Surgery, Essex Cardiothoracic Centre, Basildon, UK

${ }^{7}$ Department of Cardiac Surgery, Freeman Hospital Cardiothoracic Centre,

Newcastle upon Tyne, UK

${ }^{8}$ Department of Cardiac Surgery, Lancashire Cardiac Centre, Blackpool, UK

${ }^{9}$ Sheffield Teaching Hospitals NHS Foundation Trust Cardiothoracic Centre, Sheffield, UK

${ }^{10}$ Department of Cardiac Surgery, Aberdeen Royal Infirmary, Aberdeen, UK

${ }^{11}$ Harefield Hospital Heart Surgery, London, UK

${ }^{12}$ Department of Cardiac Surgery, Castle Hill Hospital, Cottingham, UK

${ }^{13}$ Department of Cardiac Surgery, University Hospital of Wales Healthcare NHS Trust, Cardiff, UK

${ }^{14}$ Department of Cardiac Surgery, Oxford University Hospitals NHS Trust, Oxford, UK

${ }^{15}$ Department of Cardiac Surgery, University Hospitals of Leicester NHS Trust, Leicester, UK

${ }^{16}$ Department of Cardiac Surgery, Hull University Teaching Hospitals NHS Trust, Hull, UK

${ }^{17}$ Department of Cardiac Surgery, Mater Misericordiae University Hospital, Dublin, Ireland

${ }^{18}$ Department of Cardiac Surgery, Royal Victoria Hospital, Belfast, UK

${ }^{19}$ Department of Cardiac Surgery, Liverpool Heart and Chest Hospital NHS

Foundation Trust, Liverpool, UK

${ }^{20}$ Saint Bartholomew's Hospital Barts Heart Centre, London, UK

${ }^{21}$ Department of Cardiac Surgery, Queen Elizabeth Medical Centre, Birmingham, UK

${ }^{22}$ Department of Cardiac Surgery, Southampton University Hospitals NHS Trust,

Southampton, UK

${ }^{23}$ Wythenshawe Hospital North West Heart Centre, Manchester, UK

${ }^{24}$ Department of Cardiac Surgery, University Hospital of North Staffordshire NHS

Trust, Stoke-on-Trent, UK

${ }^{25}$ Department of Cardiac Surgery, Bristol Heart Institute, Bristol, UK

${ }^{26}$ Royal Sussex County Hospital Sussex Cardiac Centre, Brighton, UK

${ }^{27}$ Department of Cardiac Surgery, Morriston Hospital, Swansea, UK

${ }^{28}$ Department of Cardiac Surgery, New Cross Hospital, Wolverhampton, UK

${ }^{29}$ Department of Cardiac Surgery, Royal Papworth Hospital NHS Foundation Trust, Cambridge, UK

${ }^{30}$ Department of Cardiac Surgery, James Cook University Hospital, Middlesbrough, UK

${ }^{31}$ Comprehensive Clinical Trials Unit, University College London Institute of Clinical Trials and Methodology, London, UK

Acknowledgements We would like to thank all the units (listed below) and the consultant surgeons who submitted their data for analysis. We are indebted to all the database managers and audit officers who have collected this data. We would like to expess our gratitude to Edwards Lifesciences for their support for a part time research fellow and administrator for this study.

Contributors The first five authors and the senior author have designed this project, compile the data set, collected, analysed the data and reviewed the various versions of the manuscript. Other authors have collated, validated and submitted their data on behalf of their unit. They have assisted in the design and analysis of the manuscript. In addition,each author has cointributed to the various verisons of the manuscipt with constructive comments. Every author has contributed to the interpretation of data. More specifically: MJah: Thought of the concept, designed the project, plan data collection, identified units, approached each unit, collected and analysed the data. Wrote the first draft and author for subsequesnt versions. RB: thought of the concept, designed the project, planned data collection, collected the data and cleaned it, contribuited significantly to writing major sections of the paper. $\mathrm{AE}-\mathrm{T}$ : Involved in the design of the study at the outset, of the two main statistician who carried out the statistical work. Attended several meetings to discuss the project, involed in writing all the versions. H-MD: Involved in the design of the study at the outset, of the two main statistician who carried out the statistical work. Attended several meetings to discuss the project, involed in writing all the versions. KM: thought of the concept, designed the project, planned data collection, collected the data and cleaned it, contribuited significantly to writing major sections of the paper, checked the literature contents and references. JA: Planned the project with the first two authors, acquired the data and contributed to several verisons during the wiriing up phase. VA: Planned the project with the first two authors, acquired the data and contributed to several verisons during the wirting up phase. MB: Planned the project with the first two authors, acquired the data and contributed to several verisons during the writing up phase. IB: Planned the project with the first two authors, acquired the data and contributed to several verisons during the writing up phase. KBo: Planned the project with the first two authors, acquired the data and contributed to several verisons during the writing up phase. NB: Planned the project with the first two authors, acquired the data and contributed to several verisons during the writing up phase. KBu: Planned the project with the first two authors, acquired the data and contributed to several verisons during the writing up phase. SB: Planned the project with the first two authors, acquired the data and contributed to several verisons during the wiriing up phase14. AC: Planned the project with the first two authors, acquired the data and contributed to several verisons during the writing up phase 15 . ID: Planned the project with the first two authors, acquired the data and contributed to several verisons during the writing up phase16. SF: Planned the project with the first two authors, acquired the data and contributed to several verisons during the writing up phase17. LH: Planned the project with the first two authors, acquired the data and contributed to several verisons during the writing up phase18. MJar: Planned the project with the first two authors, acquired the data and contributed to several verisons during the wiriing up phase19. SHJ: Planned the project with the first two authors, acquired the data and contributed to several verisons during the writing up phase20. RJ: Planned the project with the first two authors, acquired the data and contributed to several verisons during the writing up phase 9 . Inder21. MK: Planned the project with the first two authors, acquired the data and contributed to several verisons during the writing up phase22. KL: Planned the project with the first two authors, acquired the data 23. JM: Planned the project with the first two authors, acquired the data and contributed to several verisons during the writing up phase24. DM: Planned the project with the first two authors, acquired the data and contributed to several verisons during the writing up phase25. S0: Planned the project with the first two authors, acquired the data and contributed to several verisons during the writing up phase26. PP: Planned the project with the first two authors, acquired the data and contributed to several verisons during the writing up phase27. RV: Planned the project with the first two authors, acquired the data and contributed to several verisons during the wirting up phase28. PR: Planned the project with the first two authors, acquired the data and contributed to several verisons during the writing up phase29. CS: Planned the project with the first two authors, acquired the data and contributed to several verisons during the writing up phase30. SS: Planned the project with the first two authors, acquired the data and contributed to several verisons during the writing up phase31. UT: Planned the project with the first two authors, acquired the data and contributed to several verisons during the writing up phase32. AZ: Planned the project with the first two authors, acquired the data and contributed to several verisons during the writing up phase33. PY: Planned the project with the first two authors, acquired the data and contributed to several verisons during the writing up phase34. NM: Planned the project with the first two authors, acquired the data and contributed to several verisons during the writing up phase. Facilitated the acquisition of thedata through the Society for Cardiothoracic Surgery of GB \& Ireland.35. SK: Planned the project with the first two authors, acquired the data and contributed to several verisons during the writing up phase. Facilitated the acquisition of thedata through the Society for Cardiothoracic Surgery of GB \& Ireland.36. NF: Senior statistician who designed the project, defiend the original plan for the study, assisted the other two statistician to analyse the data, took part in several meetings interpreting the data with further analysis,assisted in writing the paper and final analysis

Funding We would like to expess our gratitude to Edwards Lifesciences for their support for a part time research person and administrator for this study.

Competing interests None declared.

Patient and public involvement Patients and/or the public were involved in the design, or conduct, or reporting, or dissemination plans of this research. Refer to the Methods section for further details.

Patient consent for publication Not applicable.

Ethics approval Anonymised data were submitted to the Society for Cardiothoracic Surgery of Great Britain \& Ireland (SCTS) for 27 of the 30 units and then stored in a secure database. This data are ordinarily submitted to National Institute of Cardiovascular Outcome Research (NICOR) for which local and national Caldicott guardian approvals have been obtained. A further approval from the Caldicott Guardian was obtained in 2020

Provenance and peer review Not commissioned; externally peer reviewed.

Data availability statement Data are available on reasonable request. Requests on data sharing can be made by contacting the corresponding author. Data will be shared after review and approval by the authors and terms of collaboration will be reached together with a signed data access agreement. 
Open access This is an open access article distributed in accordance with the Creative Commons Attribution Non Commercial (CC BY-NC 4.0) license, which permits others to distribute, remix, adapt, build upon this work non-commercially, and license their derivative works on different terms, provided the original work is properly cited, appropriate credit is given, any changes made indicated, and the use is non-commercial. See: http://creativecommons.org/licenses/by-nc/4.0/.

ORCID iD

Marjan Jahangiri http://orcid.org/0000-0002-4142-2889

\section{REFERENCES}

1 Zakkar M, Bryan AJ, Angelini GD. Aortic stenosis: diagnosis and management. BMJ 2016;355:i5425-9.

2 Otto CM, Burwash IG, Legget ME, et al. Prospective study of asymptomatic valvular aortic stenosis. Clinical, echocardiographic, and exercise predictors of outcome. Circulation 1997;95:2262-70.

3 Cribier A, Eltchaninoff $\mathrm{H}$, Tron C, et al. Early experience with percutaneous transcatheter implantation of heart valve prosthesis for the treatment of end-stage inoperable patients with calcific aortic stenosis. J Am Coll Cardiol 2004;43:698-703.

4 Leon MB, Smith CR, Mack M, et al. Transcatheter aortic-valve implantation for aortic stenosis in patients who cannot undergo surgery. N Engl J Med 2010;363:1597-607.

5 Adams DH, Popma JJ, Reardon MJ, et al. Transcatheter aorticvalve replacement with a self-expanding prosthesis. $N$ Engl J Med 2014;370:1790-8.

6 Mack MJ, Leon MB, Thourani VH, et al. Transcatheter aortic-valve replacement with a balloon-expandable valve in low-risk patients. $N$ Engl J Med 2019;380:1695-705.

7 Popma JJ, Deeb GM, Yakubov SJ, et al. Transcatheter aortic-valve replacement with a self-expanding valve in low-risk patients. $N$ Engl $J$ Med 2019;380:1706-15.

8 De Backer O, Luk NHV, Olsen NT, et al. Choice of Treatment for Aortic Valve Stenosis in the Era of Transcatheter Aortic Valve Replacement in Eastern Denmark (2005 to 2015). JACC Cardiovasc Interv 2016;9:1152-8.

9 Martin E, Dagenais F, Voisine P, et al. Surgical aortic valve replacement outcomes in the transcatheter era. $J$ Thorac Cardiovasc Surg 2015;150:1582-8.

10 Silaschi M, Conradi L, Treede H, et al. Trends in surgical aortic valve replacement in more than 3,000 consecutive cases in the era of transcatheter aortic valve implantations. Thorac Cardiovasc Surg 2016;64:382-9.

11 Tam DY, Rocha RV, Wijeysundera HC, et al. Surgical valve selection in the era of transcatheter aortic valve replacement in the Society of thoracic surgeons database. J Thorac Cardiovasc Surg 2020;159:416-27.

12 Simonato M, Dvir D. Transcatheter aortic valve replacement in failed surgical valves. Heart 2019;105:s38-43.

13 Landes U, Webb JG, De Backer O, et al. Repeat transcatheter aortic valve replacement for transcatheter prosthesis dysfunction. J Am Coll Cardiol 2020;75:1882-93.

14 Huygens SA, Mokhles MM, Hanif M, et al. Contemporary outcomes after surgical aortic valve replacement with bioprostheses and allografts: a systematic review and meta-analysis. Eur J Cardiothorac Surg 2016;50:605-16.

15 Thourani VH, Suri RM, Gunter RL, et al. Contemporary real-world outcomes of surgical aortic valve replacement in 141,905 lowrisk, intermediate-risk, and high-risk patients. Ann Thorac Surg 2015;99:55-61.
16 Tokuda $\mathrm{Y}$, Yamamoto $\mathrm{H}$, Miyata $\mathrm{H}$, et al. Contemporary outcomes of surgical aortic valve replacement in Japan. Circ J 2020;84:277-82.

17 Barili F, Freemantle N, Pilozzi Casado A, et al. Mortality in trials on transcatheter aortic valve implantation versus surgical aortic valve replacement: a pooled meta-analysis of Kaplan-Meier-derived individual patient data. Eur J Cardiothorac Surg 2020;58:221-9.

18 Thyregod HGH, Steinbrüchel DA, Ihlemann N, et al. Transcatheter versus surgical aortic valve replacement in patients with severe aortic valve stenosis: 1 -year results from the all-comers NOTION randomized clinical trial. J Am Coll Cardiol 2015;65:2184-94.

19 Makkar RR, Thourani VH, Mack MJ, et al. Five-Year outcomes of transcatheter or surgical aortic-valve replacement. N Engl J Med 2020;382:799-809.

20 Barbanti M, Tamburino C, D'Errigo P, et al. Five-Year outcomes of transfemoral transcatheter aortic valve replacement or surgical aortic valve replacement in a real world population. Circ Cardiovasc Interv 2019;12:1-9.

21 El Sabbagh A, Nishimura RA. Clinical conundrum of coronary artery disease and aortic valve stenosis. J Am Heart Assoc 2017;6:1-3.

22 Leon MB, Smith CR, Mack MJ, et al. Transcatheter or surgical aortic-valve replacement in intermediate-risk patients. N Engl J Med 2016;374:1609-20.

23 Sankaramangalam K, Banerjee K, Kandregula K, et al. Impact of Coronary Artery Disease on 30-Day and 1-Year Mortality in Patients Undergoing Transcatheter Aortic Valve Replacement: A MetaAnalysis. J Am Heart Assoc 2017;6:1-11.

24 Goldsweig AM, Tak HJ, Chen L-W, et al. Relative costs of surgical and transcatheter aortic valve replacement and medical therapy. Circ Cardiovasc Interv 2020;13:1-9.

25 Tripathi A, Flaherty MP, Abbott JD, et al. Comparison of causes and associated costs of 30-day readmission of transcatheter implantation versus surgical aortic valve replacement in the United States (a national readmission database study). Am J Cardiol 2018;122:431-9.

26 O'Neill A. Life expectancy (from birth) in the United Kingdom from 1765 to 2020, 2020. Available: https://www.statista.com/statistics/ 1040159/life-expectancy-united-kingdom-all-time/ [Accessed 7 Aug 2020].

27 Foroutan F, Guyatt GH, O'Brien K, O'Brien K, et al. Prognosis after surgical replacement with a bioprosthetic aortic valve in patients with severe symptomatic aortic stenosis: systematic review of observational studies. BMJ 2016;354:i5065-9

28 McClure RS, Narayanasamy N, Wiegerinck E, et al. Late outcomes for aortic valve replacement with the Carpentier-Edwards pericardial bioprosthesis: up to 17-year follow-up in 1,000 patients. Ann Thorac Surg 2010;89:1410-6.

29 Chiang YP, Chikwe J, Moskowitz AJ, et al. Survival and longterm outcomes following bioprosthetic vs mechanical aortic valve replacement in patients aged 50 to 69 years. JAMA 2014;312:1323-9.

30 Puskas JD, Gerdisch M, Nichols D, et al. Anticoagulation and Antiplatelet Strategies After On-X Mechanical Aortic Valve Replacement. J Am Coll Cardiol 2018;71:2717-26.

31 Huckaby L V, Sultan I, Gleason TG. Outcomes of tissue versus mechanical aortic valve replacement reduces long-term survival. Ann Thorac.

32 Cozijnsen L, van der Zaag-Loonen HJ, Cozijnsen MA, et al. Differences at surgery between patients with bicuspid and tricuspid aortic valves. Neth Heart J 2019;27:93-9.

33 Wallen T, Habertheuer A, Bavaria JE, et al. Elective aortic root replacement in North America: analysis of STS adult cardiac surgery database. Ann Thorac Surg 2019;107:1307-12.

34 Baumgartner H, Falk V, Bax J. 2017 ESC / EACTS guidelines for the management of valvular heart disease the task force for the management of valvular heart disease of the European Society of cardiology (ESC) and the European. Eur Heart $J$ 2017;38:2739-86. 